\title{
OPTICAL AND INFRARED SPECTROSCOPY OF CM DRACONIS
}

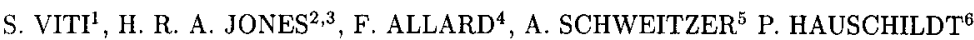 \\ ${ }^{1}$ Department of Physics and Astronomy, University College London, ${ }^{2}$ Institute of \\ Astronomy, University of Tokyo, ${ }^{3}$ Astrophysics, Liverpool JM University, ${ }^{4}$ Department \\ of Physics, Wichita State University, ${ }^{5}$ Landessternwarte Heidelberg, ${ }^{6}$ Department of \\ Physics and Astronomy, The University of Georgia
}

\section{Abstract}

We compare observations of the binary system CM Draconis with synthetic spectra. Spectroscopic observations from 0.40 to $2.41 \mu \mathrm{m}$, combined with photometry and the accurately known surface gravity enable us to estimate the temperature and metallicity using detailed spectra synthesis. We find discrepancies between the analysis of the infrared and optical spectrum: while the optical spectral energy distribution (SED) yields a metal-rich solution with $T_{\text {eff }}=3000 \mathrm{~K}$, the infrared SED yields around $3200 \mathrm{~K}$ and $-0.8 \leq[\mathrm{M} / \mathrm{H}] \leq-0.6$ compatible with the high space motion of the system. The low-metallicity characteristics of the infrared SED could be real and is partly supported by the detailed analysis of the atomic lines in the optical region. Although, the known incompleteness of the $\mathrm{TiO}$ and $\mathrm{H}_{2} \mathrm{O}$ line lists in the models may cause substantial systematic errors.

\section{Background}

CM Draconis (hereafter, CM Dra) is the lowest mass main-sequence eclipsing binary known. It has a short period of 1.268 days (Metcalfe et al. 1996, ApJ, 456, 356), a very large space velocity (163 $\mathrm{km} \mathrm{s}^{-1}$ ) and low flaring rate which suggests it belongs to Population II (Lacy 1977, ApJ, $218,444)$. Because it is one of the faintest, smallest and least massive main-sequence binaries so far known, it is a prime calibrator of what happens to very low mass stars at the bottom of the main sequence. Some of the fundamental physical properties of the components of CM Dra have already been accurately determined. Most important are the individual masses of $M_{\mathrm{A}}=0.2307 \pm$ 0.0010 and $M_{\mathrm{B}}=0.2136 \pm 0.0010 M_{\odot}$ and radii of $R_{\mathrm{A}}=0.2516 \pm 0.0020$ and $R_{\mathrm{B}}=0.2347 \pm 0.0019$ $R_{\odot}$ (Metcalfe et al., 1996), and surface gravities of $\log g=4.999 \pm 0.007$ for CM Dra A and log $g=5.028 \pm 0.007$ for CM Dra B (Jones et al. 1996, MNRAS, 277, 767). The precision of these values far exceeds those known for any $\mathrm{M}$ dwarf and means that $\mathrm{CM}$ Dra is an excellent system for comparison with model calculations. The spectra taken for our study are shown below in Figure 1.
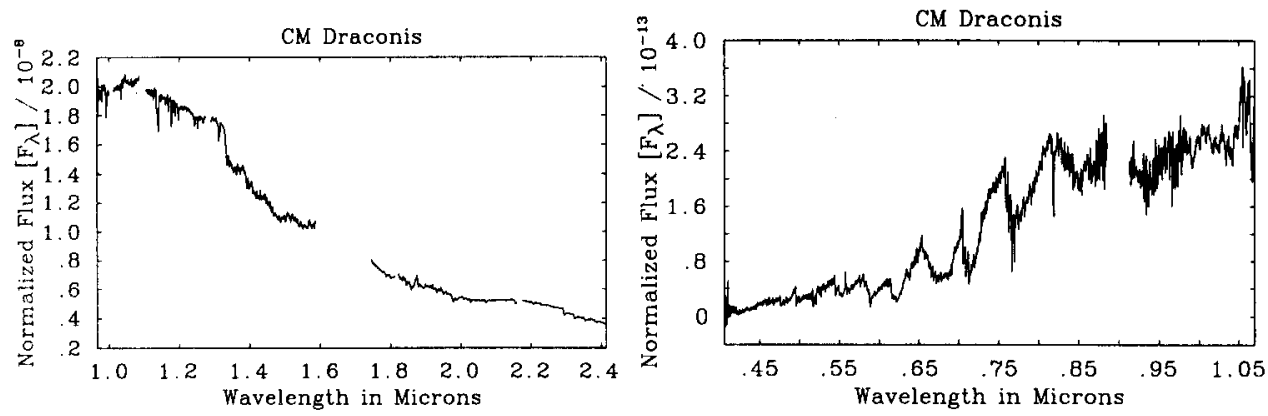

Figure 1. (a) Infrared spectrum and (b) optical spectrum of CM Dra from 0.4056 to $2.41 \mu \mathrm{m}$. 